\title{
EFEITO DE FOSFATOS NATURAIS EM PLANTAS DE MELÃO CULTIVADAS EM VASOS ${ }^{(1)}$
}

\author{
Clementino Marcos Batista de Faria ${ }^{(2)}$, Davi José Silva ${ }^{(2)}$, José Maria \\ Pinto $^{(2)}$ \& Tâmara Claudia de Araújo Gomes ${ }^{(2)}$
}

\begin{abstract}
RESUMO
Com o advento da agricultura orgânica no Vale do Submédio São Francisco, surge a demanda por técnicas que atendam aos princípios desse sistema de exploração agrícola. Dessa forma, realizou-se este trabalho com o objetivo de avaliar a eficiência de fosfatos naturais em três solos (Argissolo Acinzentado distrófico PACd, Argissolo Amarelo eutrófico - PAe e Vertissolo - V) da região, cultivados com melão (Cucumis melo) em vasos, na Embrapa Semi-Árido. Os tratamentos constituíram um fatorial $(3 \times 4)+1$, sendo três doses de $P\left(40,80\right.$ e $160 \mathrm{mg} \mathrm{dm}^{-3} \mathrm{de}$ $\mathrm{P}_{2} \mathrm{O}_{5}$ ), quatro fontes de $\mathrm{P}$ (superfosfato triplo-ST, termofosfato-TM, fosfato natural de Gafsa-FG e fosfato natural Fosbahia-FB) e uma testemunha sem $P$, dispostos no delineamento experimental inteiramente casualizado, com três repetições. Cada solo constituiu um ensaio. Após 38 dias de cultivo do melão, avaliaram-se a produção de matéria seca (MS) da parte aérea, o $\mathrm{P}$ absorvido pela planta e o $\mathrm{P}$ do solo extraído pelos métodos de Mehlich-1 e resina de troca aniônica (RTA). No V e PACd, os fosfatos apresentaram-se menos eficientes que no PAe. O TM foi a fonte de $P$ que apresentou maior eficiência para produção de MS do melão nos três solos (56 a 100 \% em relação ao superfosfato triplo). No PAe, o FG também mostrou boa eficiência, sendo equivalente a 80 \% do superfosfato triplo. Os extratores Mehlich e RTA foram igualmente eficientes para avaliar a disponibilidade do $P$.
\end{abstract}

Termos de indexação: Cucumis melo, solo, fósforo, extrator químico.

(1) Trabalho financiado com recursos do PRODETAB. Recebido para publicação em maio de 2004 e aprovado em novembro de 2006.

(2) Pesquisador da Embrapa Semi-Árido. Caixa Postal 23, CEP 56302-970 Petrolina (PE). E-mails: clementino.faria@ig.com.br; davi@cpatsa.embrapa.br; jmpinto@cpatsa.embrapa.br; tamara@cpatc.embrapa.br 


\title{
SUMMARY: EFFECT OF NATURAL PHOSPHATES ON MELON PLANTS CULTIVATED IN POTS
}

\begin{abstract}
Organic agriculture is an emerging activity in the upper mid São Francisco River Valley, requiring technologies that are in line with the principles of this agricultural system. A study was carried out at Embrapa Semi-Árido to evaluate the efficacy of natural phosphates applied to three regional soils (dystrophic Grey Argisol - PAGd, eutrophic Yellow Argisol - PAe and Vertisol - V) planted with melon (Cucumis melo) in pots accommodated on wooden benches. The factorial treatments $(3 \times 4)+1$ consisted of three phosphorus levels (40, 80 and $160 \mathrm{mg} \mathrm{dm}^{-3} \mathrm{P}_{2} \mathrm{O}_{5}$ ), four phosphorus sources (triple superphosphate-ST, thermophosphate-TM, Gafsa natural rock phosphate-FG and Fosbahia natural phosphate$F B$ ) and a control treatment without phosphorus, in a completely randomized design with three replications. Each soil represented a trial. Shoot dry matter (DM), P plant content and $P$ soil concentration extracted by Mehlich-1 and anionic exchange resin (AER) were evaluated 38 days after planting. In $V$ and PAGd soils the phosphates were less effective than in PAe soil. TM was the P source with the highest efficiency for DM production in the three soils (56 to $100 \%$ in relation to triple super phosphate). In PAe, FG also presented relatively high efficiency, equivalent to $80 \%$ of that obtained with triple super phosphate. The Mehlich-1 and AER extractors were equally effective at evaluating $P$ availability.
\end{abstract}

Index terms: Cucumis melo, soil, phosphorus, chemical extractor.

\section{INTRODUÇÃO}

Recentemente, os produtores do Vale do Submédio São Francisco, incentivados pelo mercado de frutas, têm apresentado, de forma crescente, demandas por técnicas para produção orgânica. Considerando que os princípios da agricultura orgânica não permitem o uso dos fertilizantes solúveis, oriundos de tratamento químico, os fosfatos naturais (FN) e os fosfatos tratados termicamente são as opções para serem usadas nas adubações fosfatadas nesse sistema. Entretanto, a eficiência dos FN depende muito de suas próprias características, incluindo sua origem, das propriedades do solo, da forma como são usados e das características da planta a ser cultivada (Chien \& Menon, 1995; Novais \& Smyth, 1999).

Os FN do Brasil, por não serem de origem sedimentar, têm, geralmente, apresentado resultados de baixa eficiência agronômica (Goedert \& Lobato, 1980; Yost et al., 1982; Oliveira et al.,1984; Goedert \& Lobato, 1984; León et al., 1986; Sanzonowicz \& Goedert, 1986; Sanzonowicz et al., 1987). Os fosfatos considerados reativos (teor de $\mathrm{P}_{2} \mathrm{O}_{5}$ solúvel em ácido cítrico igual ou superior a $30 \%$ do teor total de $\mathrm{P}_{2} \mathrm{O}_{5}$ ), como os FN Gafsa, da Tunísia, Arad, de Israel e Carolina do Norte, dos Estados Unidos, e os termofosfatos apresentam resultados semelhantes aos fosfatos solúveis (Yost et al., 1982; Oliveira et al., 1984; Goedert \& Lobato, 1984; Sanzonowicz et al., 1987; Braga et al., 1991). A granulometria dos FN também pode influir na sua eficiência (Vasconcelos et al., 1986a; Chien \& Menon, 1995).

$\mathrm{O}$ Ca e o $\mathrm{P}$ liberados dos fertilizantes fosfatados para a solução do solo são, em grande parte, absorvidos pela planta e adsorvidos pelo próprio solo. Portanto, o solo e a planta funcionam como drenos para Ca e P. Solos ácidos, pobres em bases e em $\mathrm{P}$, com predominância de cargas positivas (liberação alta de prótons $-\mathrm{H}^{+}$), com CTC elevada (grande "dreno" de Ca) e, ao mesmo tempo, com baixo poder tampão de $\mathrm{P}$, oferecem melhores condições para que o uso dos FN seja mais eficiente (Novais \& Smyth, 1999).

Os principais solos irrigáveis do Submédio São Francisco são os Latossolos e os Argissolos, ambos de textura arenosa, com acidez leve e baixa CTC e pobres em P; os Luvissolos, de textura média, com acidez quase neutra e valores de CTC e teores de P médios, e os Vertissolos, de textura argilosa, reação alcalina, com alta CTC e pobres em $\mathrm{P}(\mathrm{FAO}, 1966)$. A capacidade máxima de sorção de fosfato desses solos varia de 0,124 a $0,636 \mathrm{mg} \mathrm{g}^{-1}$ de P (Pereira \& Faria, 1998).

As plantas que promovem abaixamento do $\mathrm{pH}$ na rizosfera pela extrusão de prótons de suas raízes e que absorvem muito $\mathrm{Ca}$ (dreno de $\mathrm{Ca}$ ) são as mais eficientes para utilização do P oriundo dos FN (Novais $\&$ Smyth, 1999). Zoysa (1999) atribuiu ao decréscimo do $\mathrm{pH}$ na zona da rizosfera de plantas de chá o resultado positivo do uso de um fosfato de rocha em comparação com o superfosfato triplo.

O meloeiro, que é uma das hortaliças mais cultivadas no Vale do Submédio São Francisco, é considerado exigente em $\mathrm{Ca}$. Com uma produtividade média de 31,5 $\mathrm{t} \mathrm{ha}^{-1}$ de melão entre os oito híbridos avaliados, a cultura extraiu 80,6 $\mathrm{kg} \mathrm{ha}^{-1} \mathrm{de} \mathrm{Ca}$ (Lima, 2001). No trabalho realizado por Prata (1999), o Ca foi o nutriente absorvido e exportado em maior quantidade. Com relação à exigência em $\mathrm{P}, 116 \mathrm{~kg} \mathrm{ha}^{-1}$ de $\mathrm{P}_{2} \mathrm{O}_{5}$ foi a dose que proporcionou a produtividade máxima esperada $\left(29,1 \mathrm{t} \mathrm{ha}^{-1}\right)$ num Vertissolo dessa região (Faria et al., 1994). 
Nos trabalhos de avaliação dos FN como fonte de $\mathrm{P}$, têm sido usados o índice de eficiência agronômica (IEA) e o equivalente a superfosfato triplo (EqST) para medir sua eficiência (Sanzonowicz \& Goedert, 1986; Almeida et al., 1996). Quando se dispõe de uma curva de resposta da planta a aplicações do superfosfato triplo, é preferível usar o EqST (Goedert \& Lobato, 1984), escolhendo-se a dose de $\mathrm{P}_{2} \mathrm{O}_{5}$ que proporcione um rendimento em torno de 80 \% da produção máxima (Sanzonowicz \& Goedert, 1986). Dos extratores usados para avaliar a disponibilidade de P no solo, o Mehlich-1 superestima a quantidade do $\mathrm{P}$ disponibilizada dos $\mathrm{FN}$ aplicados no solo (Yost et al., 1982; Almeida et al., 1996), sendo a resina de troca aniônica (RTA) mais apropriada para essa finalidade (Braga et al., 1991; Mutuo, 1999; Silva \& Raij, 1999). Entretanto, algumas pesquisas têm mostrado que o P-Mehlich-1 apresentou altas correlações com o $\mathrm{P}$ absorvido pela planta e o P-RTA (Vasconcelos et al., 1986b; Freitas et al., 1995; Moreira \& Malavolta, 2001).

Este trabalho foi realizado com o objetivo de avaliar a eficiência de fosfatos naturais no cultivo do melão em experimento em vasos.

\section{MATERIAL E MÉTODOS}

O trabalho foi realizado em vasos, na Embrapa Semi-Árido, em Petrolina (PE), de outubro a dezembro de 2002, com três tipos de solo, Argissolo Acinzentado distrófico, Argissolo Amarelo eutrófico e Vertissolo
(Embrapa, 1999) (Quadro 1). As amostras de solo foram coletadas na camada de $0-20 \mathrm{~cm}$, sendo os dois primeiros do município de Petrolina (PE) e o terceiro de Juazeiro (BA). Os tratamentos constituíram um fatorial $(3 \times 4)+1$, sendo três doses de $\mathrm{P}(40,80 \mathrm{e}$ $160 \mathrm{mg} \mathrm{dm}^{-3}$ de $\mathrm{P}_{2} \mathrm{O}_{5}$ ), quatro fontes de $\mathrm{P}$ (superfosfato triplo, termofosfato, fosfato natural de Gafsa da Tunísia e fosfato natural Fosbahia (Quadro 2)) e uma testemunha sem $\mathrm{P}$, dispostos no delineamento experimental inteiramente casualizado, com três repetições. Cada solo constituiu um ensaio. O superfosfato triplo foi masserado de tal forma, que suas partículas tornaram-se $30 \%$ menores que 0,297 mm, antes de ser usado.

A unidade experimental foi constituída de um vaso com $3 \mathrm{dm}^{3}$ de solo, com duas plantas de melão (Cucumis melo), cultivar AF-682. Em 18 de outubro de 2002, foi feita a calagem no Argissolo Acinzentado com $750 \mathrm{mg} \mathrm{dm}^{-3}\left(1,5 \mathrm{t} \mathrm{ha}^{-1}\right)$ de calcário com PRNT $115 \%$, que continha $64,0 \%$ de $\mathrm{CaO}$ e $0,5 \%$ de $\mathrm{MgO}$, mantendo-se o solo úmido na capacidade de campo, por 20 dias. Os demais solos não receberam calagem. Em 11 de novembro de 2002, aplicou-se o P, misturando-se o fosfato com todo volume do solo de cada vaso e, em seguida, realizou-se o plantio do melão. A irrigação foi feita diariamente, por gotejamento, utilizando-se emissor com vazão de $2,4 \mathrm{~L} \mathrm{~h}^{-1}$, sendo a lâmina aplicada com base no coeficiente de cultivo (Kc) e na evaporação do tanque classe $\mathrm{A}$. O volume de água aplicado foi de, aproximadamente, $0,8 \mathrm{~L}$ por planta em uma irrigação por dia, até 30 dias após o plantio, e depois, 1,4 L por planta, parcelado em duas irrigações diárias.

Quadro 1. Características físicas e químicas de amostras dos solos coletadas na camada de 0-20 cm antes da aplicação de corretivo e dos fertilizantes utilizados no experimento ${ }^{(1)}$

\begin{tabular}{|c|c|c|c|}
\hline \multirow{2}{*}{ Característica } & \multicolumn{3}{|c|}{ Solo } \\
\hline & Argissolo Acinzentado & Argissolo Amarelo & Vertissolo \\
\hline Areia $\left(\mathrm{g} \mathrm{kg}^{-1}\right)$ & 90 & 84 & 24 \\
\hline Silte $\left(\mathrm{g} \mathrm{kg}^{-1}\right)$ & 6 & 10 & 23 \\
\hline Argila (g kg-1) & 4 & 6 & 53 \\
\hline $\mathrm{pH}$ em $\mathrm{H}_{2} \mathrm{O}(1: 2,5)$ & 4,9 & 5,9 & 7,3 \\
\hline $\mathrm{CEe}\left(\mathrm{dS} \mathrm{m}^{-1}\right)$ & 0,12 & 0,12 & 0,18 \\
\hline $\mathrm{Ca}^{2+}\left(\mathrm{cmol}_{\mathrm{c}} \mathrm{dm}^{-3}\right)$ & 0,2 & 1,5 & 19,8 \\
\hline $\mathrm{Mg}^{2+}\left(\mathrm{cmol}_{\mathrm{c}} \mathrm{dm}^{-3}\right)$ & 0,3 & 0,7 & 3,5 \\
\hline $\mathrm{K}^{+}\left(\mathrm{cmol}_{\mathrm{c}} \mathrm{dm}^{-3}\right)$ & 0,10 & 0,28 & 0,32 \\
\hline $\mathrm{Na}^{+}\left(\mathrm{cmol}_{\mathrm{c}} \mathrm{dm}^{-3}\right)$ & 0,02 & 0,02 & 0,07 \\
\hline $\mathrm{Al}^{3+}\left(\mathrm{cmol}_{\mathrm{c}} \mathrm{dm}^{-3}\right)$ & 0,50 & 0,05 & 0,00 \\
\hline $\mathrm{H}+\mathrm{Al}\left(\mathrm{cmol}_{\mathrm{c}} \mathrm{dm}^{-3}\right)$ & 1,92 & 0,76 & 0,00 \\
\hline CTC pH 7,0 $\left(\mathrm{cmol}_{\mathrm{c}} \mathrm{dm}^{-3}\right)$ & 2,54 & 3,26 & 23,69 \\
\hline $\mathrm{V}(\%)$ & 24 & 77 & 100 \\
\hline $\mathrm{P}\left(\mathrm{mg} \mathrm{dm} \mathrm{m}^{-3}\right)$ & 2 & 2 & 2 \\
\hline M.O. $\left(\mathrm{g} \mathrm{kg}^{-1}\right)$ & 7,9 & 7,7 & 12,2 \\
\hline
\end{tabular}

(1) Embrapa (1997). 
Quadro 2. Características físicas e químicas dos fosfatos avaliados

\begin{tabular}{|c|c|c|c|c|}
\hline \multirow[b]{2}{*}{ Fosfato } & \multicolumn{2}{|c|}{$\mathrm{P}_{2} \mathrm{O}_{5}$ solúvel } & \multirow{2}{*}{$\begin{array}{l}\mathrm{P}_{2} \mathrm{O}_{5} \text { ácido } \\
\text { cítrico/total }\end{array}$} & \multirow{2}{*}{$\begin{array}{l}\text { Partículas retidas } \\
\text { em peneira de } 50 \text { mesh }\end{array}$} \\
\hline & Ácido cítrico $2 \%$ & Total & & \\
\hline & $\%$ & $\underline{-}$ & & $\%$ \\
\hline Superfosfato triplo ${ }^{(1)}$ & 46,4 & 46,4 & 100 & 70,2 \\
\hline Termofosfato & 16,3 & 16,5 & 98 & 3,7 \\
\hline Gafsa & 11,5 & 25,6 & 45 & 24,1 \\
\hline Fosbahia & 5,8 & 26,0 & 22 & 3,0 \\
\hline
\end{tabular}

Dois dias após a emergência das plantas, todos os tratamentos receberam uma adubação, via solução, correspondente às quantidades, em $\mathrm{mg} \mathrm{dm}^{-3}$, de $25 \mathrm{de}$ $\mathrm{N}, 75$ de $\mathrm{K}_{2} \mathrm{O}, 2,5$ de $\mathrm{Zn}, 0,75$ de $\mathrm{B}$ e 0,25 de Mo, sob as formas de uréia, sulfato de potássio, sulfato de zinco, ácido bórico e molibdato de sódio, respectivamente. Posteriormente, foram aplicados $25 \mathrm{mg} \mathrm{dm}^{-3} \mathrm{de} \mathrm{N} \mathrm{na}$ forma de uréia, via solução, para todos os tratamentos aos 11 e 22 dias do plantio.

Algum excesso de água proveniente da irrigação era drenado por meio de furos no fundo dos vasos. Com isso, é possível que tenha havido alguma perda de nutrientes, principalmente de $\mathrm{N}$, por ser mais móvel no solo, mas não se observou sintoma de deficiência nas plantas de melão.

Aos 38 dias do plantio, procedeu-se ao corte da parte aérea do melão, que foi acondicionada em sacos de papel e colocada numa estufa com circulação de ar, para secar sob temperatura de $65^{\circ} \mathrm{C}$, por três dias, a fim de se obter a massa seca (MS). Depois, retirou-se uma amostra de cada unidade experimental para determinação do teor de $\mathrm{P}$ na planta, segundo Malavolta et al. (1997). Em seguida, foi feita uma amostragem no solo para determinações de $\mathrm{P}$ pelos extratores de Mehlich-1 (Embrapa, 1997) e resina de troca aniônica (Raij et al., 2001). Os dados obtidos foram submetidos a análises estatísticas de variância, teste de médias e regressão, segundo Snedecor \& Cochran (1971). Quando o teste F era significativo nas análises de regressão, realizadas com os dados referentes à dose de $\mathrm{P}$, procurava-se ajustar equações lineares ou quadráticas da produção de MS, absorção de $\mathrm{P}$ pela parte aérea do melão, o $\mathrm{P}$ Mehlich-1 e o P-resina de acordo com as doses 0, 40, 80 e $160 \mathrm{mg} \mathrm{dm}^{-3} \mathrm{de}_{2} \mathrm{O}_{5}$; e do $\mathrm{P}$ absorvido pelo melão, considerando o $\mathrm{P}$ recuperado do solo por esses dois extratores químicos.

\section{RESULTADOS E DISCUSSÃO}

O meloeiro respondeu positivamente ao $\mathrm{P}$ na forma de superfosfato triplo aplicado nos três solos (Figura 1), cujas doses que proporcionaram a produção máxima estimada de matéria seca (MS) da parte aérea (Quadro 3) foram de 98, 133 e $142 \mathrm{mg}$ vaso $^{-1}$ de $\mathrm{P}_{2} \mathrm{O}_{5}$, respectivamente, no Argissolo Acinzentado distrófico (PACd), Argissolo Amarelo (PAe) e Vertissolo (V). Este último solo, com teor elevado de argila (Quadro 1), apresenta também maior capacidade de adsorção de P (Pereira \& Faria, 1998), exigindo, assim, maior quantidade de $\mathrm{P}_{2} \mathrm{O}_{5}$ para se obter a produção máxima.

Para as outras fontes de $\mathrm{P}$, que, na análise de variância, o teste $\mathrm{F}$ foi significativo, observou-se efeito quadrático também do termofosfato no PACd e PAe e
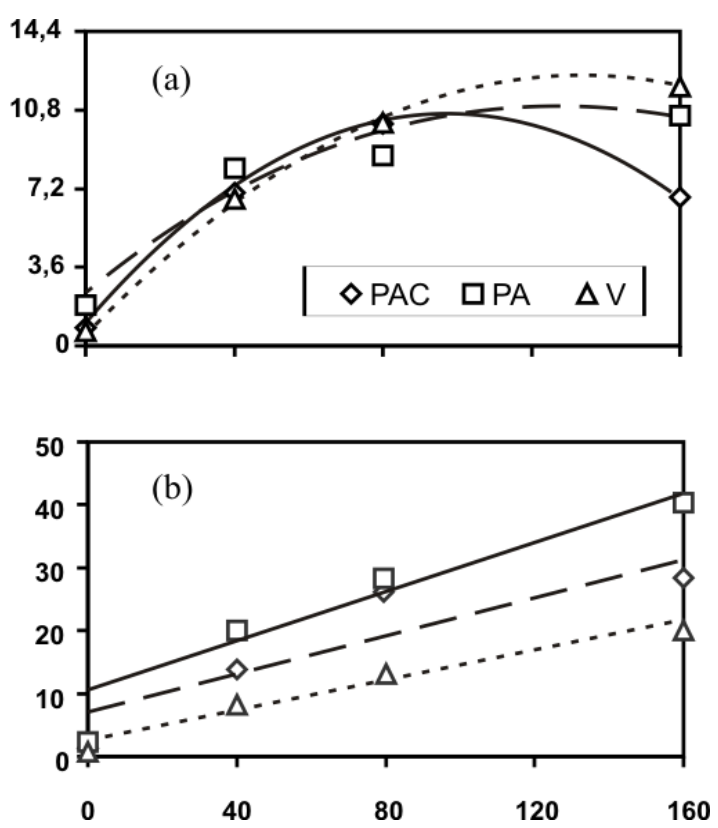

Figura 1. Relações entre as doses de fósforo na forma de superfosfato triplo aplicadas no Argissolo Acinzentado (PAC), Argissolo Amarelo (PA) e Vertissolo (V) e a produção de matéria seca (MS) do melão (a) e o fósforo absorvido pelas plantas (b). 
do fosfato de Gafsa no PAe. Ainda houve efeito linear do fosfato Fosbahia no PAe e do termofosfato no V. No PACd e no V, o Gafsa e o Fosbahia não exerceram efeito significativo (Quadro 3). Efeitos semelhantes foram observados para a absorção de P pela parte aérea do melão, porém com respostas lineares (Figura 1), demonstrando que a absorção de P pelo melão ainda continua crescente, mesmo quando a produção de matéria seca já tenha atingido o valor máximo. No $\mathrm{PAe}$, as quantidades de $\mathrm{P}$ absorvidas pelo melão, quando foram aplicados os fosfatos de Gafsa e Fosbahia, foram menores que as do superfosfato triplo e do termofosfato, o que se refletiu na produção de matéria seca (Quadros 3 e 4).

Observa-se que a absorção de $\mathrm{P}$ pelo melão, aplicado na forma de superfosfato triplo e de termofosfato, foi menor no Vertissolo que nos outros dois solos (Quadro 4), o que pode estar relacionado com a capacidade máxima de adsorção de $\mathrm{P}$ nos três solos estudados. Enquanto, no PACd e PAe, esses valores variaram de 0,124 a $0,138 \mathrm{mg} \mathrm{dm}^{-3} \mathrm{de} \mathrm{P}$, no Vertissolo, foi de $0,528 \mathrm{mg} \mathrm{dm}^{-3}$ de $\mathrm{P}$, segundo Pereira \& Faria (1998). Galrão \& Volkweiss (1981) observaram que, quanto maior o poder tampão de $\mathrm{P}$, menor é a absorção de $\mathrm{P}$ por unidade de $\mathrm{P}$ aplicado. Em ensaios com soja, os solos que apresentaram maior capacidade tampão de fosfato proporcionaram menor absorção e acúmulo de P na planta, contribuindo para maior eficiência na sua utilização (Muniz et al., 1985; Bedin et al., 2003).

Utilizou-se a dose de $80 \mathrm{mg} \mathrm{dm}^{-3}$, que está numa posição ascendente da curva de produção de MS (Figura 1), para calcular o equivalente ao superfosfato triplo (EqST), conforme recomendação de Sanzonowicz \& Goedert (1986) (Quadro 4). O termofosfato estatisticamente não diferiu do superfosfato triplo nos dois Argissolos, com índices de 74,3 e 100,1 \% de EqST, para o PACd e PAe, respectivamente, e inferior no Vertissolo, cujo EqST foi de 55,9\%. Os fosfatos de Gafsa e o Fosbahia foram significativamente inferiores às outras duas fontes de $\mathrm{P}$ nos três solos, sendo, no PAe o Gafsa, com EqST de 80,0 \%, superior ao fosbahia, com EqST de 38,4 \%. Nos outros dois solos, esses fosfatos apresentaram EqSTs muito baixos, de 9,5 a $10,2 \%$.

Esses resultados evidenciam que o termofosfato pode ser usado no cultivo orgânico do melão com alta eficiência no PAe, eficiência média no PACd e com menor eficiência no Vertissolo. Em trabalho realizado em PAe com milho nessa região, Pereira \& Faria (1988) verificaram que alguns fosfatos naturais (FN) brasileiros, quando tratados termicamente, apresentaram índice de equivalência agronômica de 84 a $105 \%$. O Vertissolo, por sua própria constituição química (elevado $\mathrm{pH}$ e alto teor de Ca), limita a solubilidade dos FN (Chien \& Menon, 1995; Novais \& Smyth, 1999). O PACd é o solo que tem o pH mais baixo, o que favorece a solubilização do FN. Porém, por ter recebido calagem e apresentar CTC muito baixa (Quadro 1) e, conseqüentemente, poucos "drenos" para adsorver os íons de Ca liberados dos FN, esse solo dificulta a continuidade da solubilização, contribuindo para diminuir a eficiência dos FN (Chien $\&$ Menon, 1995).

Os fosfatos de Gafsa e Fosbahia apresentaram teores de $\mathrm{P}_{2} \mathrm{O}_{5}$ total semelhantes e mais elevados que o do termofosfato, porém teores de $\mathrm{P}_{2} \mathrm{O}_{5}$ solúvel em ácido cítrico a $2 \%$ e valores da reatividade (relação Pácido cítrico/P-total) inferiores aos do termofosfato (Quadro 2), o que explica a superioridade deste último sobre os dois primeiros, considerando que existe

Quadro 3. Equações de regressão para produção de matéria seca (MS) e absorção de fósforo pela parte aérea do melão, considerando as doses de fósforo $\left(\mathrm{mg} \mathrm{dm}^{-3} \mathrm{de}_{2} \mathrm{O}_{5}\right)$ aplicadas nos diferentes solos, na forma de diferentes fosfatos

\begin{tabular}{|c|c|c|c|c|c|c|}
\hline \multirow[b]{2}{*}{ Solo(1) } & \multirow[b]{2}{*}{ Fosfato } & \multicolumn{2}{|c|}{ MS do melão (g/vaso) } & \multicolumn{3}{|c|}{ Absorção de P (mg/vaso) } \\
\hline & & Equação & $\mathbf{r}^{2}$ & & Equação & $\mathbf{r}^{2}$ \\
\hline \multirow[t]{2}{*}{ PACd } & S. triplo & $\hat{y}=0,8+0,196 x-0,0010 x^{2}$ & 0,99 & $\hat{y}$ & $=4,8+0,168 x$ & 0,86 \\
\hline & Termofosfato & $\hat{y}=1,3+0,135 x-0,0006 x^{2}$ & 0,93 & $\hat{y}$ & $=2,7+0,207 x$ & 0,98 \\
\hline \multirow[t]{4}{*}{ PAe } & S. triplo & $\hat{y}=2,3+0,133 x-0,0005 x^{2}$ & 0,93 & $\hat{y}$ & $=5,4+0,231 x$ & 0,96 \\
\hline & Termofosfato & $\hat{y}=2,5+0,142 x-0,0006 x^{2}$ & 0,86 & $\hat{y}$ & $=6,2+0,140 \mathrm{x}$ & 0,88 \\
\hline & Gafsa & $\hat{y}=1,7+0,094 x-0,0004 x^{2}$ & 0,98 & $\hat{y}$ & $=4,1+0,077 \mathrm{x}$ & 0,85 \\
\hline & Fosbahia & $\hat{y}=1,8+0,017 x$ & 0,97 & $\hat{y}$ & $=2,0+0,032 \mathrm{x}$ & 0,98 \\
\hline \multirow[t]{2}{*}{$\mathrm{V}$} & S. triplo & $\hat{y}=0,7+0,170 x-0,0006 x^{2}$ & 0,99 & $\hat{y}$ & $=2,3+0,117 \mathrm{x}$ & 0,97 \\
\hline & Termofosfato & $\hat{y}=0,9+0,058 x$ & 0,99 & $\hat{y}$ & $=0,1+0,063 \mathrm{x}$ & 0,70 \\
\hline
\end{tabular}

(1) PACd: Argissolo Acinzentado distrófico; PAe: Argissolo Amarelo eutrófico; V: Vertissolo. 
correlação positiva entre o $\mathrm{P}$ solúvel em ácido cítrico do FN e sua eficiência (Oliveira et al., 1984; Vasconcelos et al., 1986a; Sanzonowicz \& Goedert, 1986). O Fosbahia, mesmo apresentando granulometria mais fina que a do Gafsa (Quadro 2), por ser menos reativo que este, revelou menor eficiência que a do Gafsa, no PAe (Quadro 4). Syers et al. (1986) mencionaram que a eficiência dos FN depende mais de sua solubilidade nos extratores químicos que da sua área de superfície específica. A solubilidade de fosfatos naturais em ácido cítrico $2 \%$ tem sido um critério adequado para avaliação de sua eficiência agronômica, existindo correlação altamente significativa entre estas características (Oliveira et al., 1984).
Observou-se boa correlação entre o P absorvido pela parte aérea do melão e o $\mathrm{P}$ extraído do solo pelos métodos Mehlich-1 e resina de troca aniônica, nos casos em que houve efeito significativo da aplicação de $\mathrm{P}$ na produção de MS e na absorção do $\mathrm{P}$ pelo melão (Quadro 5). Apenas para o P extraído por Mehlich-1 nos tratamentos com termofosfato no $\mathrm{PAe}$, o coeficiente de determinação $\left(R^{2}\right)$ foi inferior a 0,90 . A correlação linear obtida com os dois extratores para o $\mathrm{P}$ do fosbahia no PAe demonstra a necessidade de serem usadas doses mais elevadas de $\mathrm{P}$ com vistas em atingir absorção máxima, demonstrando a baixa eficiência desse fosfato. No Vertissolo, o P extraído por Mehlich-1 do termofosfato também proporcionou efeito linear. Para

Quadro 4. Média da produção de matéria seca (MS) da parte aérea do melão obtida com os diferentes fosfatos e a equivalência ao superfosfato triplo (EqSt) de cada fosfato, quando empregado na dose de $80 \mathrm{mg} \mathrm{dm}^{-3}$ de $\mathrm{P}_{2} \mathrm{O}_{5}$ nos três solos

\begin{tabular}{|c|c|c|c|c|c|c|}
\hline \multirow{3}{*}{ Fosfato $^{(1)}$} & \multicolumn{6}{|c|}{ Solo(1) } \\
\hline & \multicolumn{2}{|c|}{ Argissolo Acinzentado } & \multicolumn{2}{|c|}{ Argissolo Amarelo } & \multicolumn{2}{|c|}{ Vertissolo } \\
\hline & MS & EqSt & MS & EqSt & MS & EqSt \\
\hline & $\mathrm{g} /$ vaso & $\%$ & g/vaso & $\%$ & $\mathrm{~g} /$ vaso & $\%$ \\
\hline Superfosfato triplo & $8,00 \mathrm{a}$ & 100,0 & $9,12 \mathrm{a}$ & 100,0 & $9,59 \mathrm{a}$ & 100,0 \\
\hline Termofosfato & $7,78 \mathrm{a}$ & 74,3 & $9,33 \mathrm{a}$ & 100,1 & $6,49 \mathrm{~b}$ & 55,9 \\
\hline Gafsa & $0,93 \mathrm{~b}$ & 9,6 & $5,97 \mathrm{~b}$ & 80,0 & $0,84 \mathrm{c}$ & 9,5 \\
\hline Fosbahia & $0,95 \mathrm{~b}$ & 10,2 & $3,37 \mathrm{c}$ & 38,4 & $1,00 \mathrm{c}$ & 9,7 \\
\hline CV (\%) & 25,8 & - & 16,6 & - & 14,6 & - \\
\hline
\end{tabular}

(1) Valores seguidos da mesma letra, na mesma coluna, não diferem significativamente pelo teste Duncan a $5 \%$.

Quadro 5. Regressão entre a concentração de fósforo no solo pelos extratores Melhich-1 e resina de troca aniônica ( $\mathrm{mg} \mathrm{dm}^{-3}$ de $\left.\mathrm{P}\right)$ e a absorção do nutriente $\left(\mathrm{mg}\right.$ vaso $\left.^{-1} \mathrm{de} \mathrm{P}\right)$ pelo melão ocorrida nos solos, com os diferentes fosfatos

\begin{tabular}{|c|c|c|c|c|c|c|}
\hline \multirow[b]{2}{*}{ Solo(1) } & \multirow[b]{2}{*}{ Fosfato } & \multicolumn{2}{|l|}{ P-Mehlich-1 } & \multicolumn{3}{|c|}{ P-resina } \\
\hline & & Equação & $\mathbf{R}^{2}$ & & Equação & $\mathbf{R}^{2}$ \\
\hline \multirow[t]{2}{*}{ PACd } & S. triplo & $\hat{y}=6,4-3,093 x-0,0682 x^{2}$ & 0,99 & $\hat{y}$ & $=8,8-4,887 \mathrm{x}-0,1559 \mathrm{x}^{2}$ & 0,97 \\
\hline & Termofosfato & $\hat{y}=3,9-2,046 x-0,0268 x^{2}$ & 0,99 & $\hat{y}$ & $=5,7-3,806 \mathrm{x}-0,0868 \mathrm{x}^{2}$ & 0,99 \\
\hline \multirow[t]{4}{*}{ PAe } & S. triplo & $\hat{y}=13,4-4,984 \mathrm{x}-0,1152 \mathrm{x}^{2}$ & 0,99 & $\hat{y}$ & $=25,4-7,731 \mathrm{x}-0,2221 \mathrm{x}^{2}$ & 0,99 \\
\hline & Termofosfato & $\hat{y}=2,6-2,403 x-0,0478 x^{2}$ & 0,89 & $\hat{y}$ & $=19,3-6,351 \mathrm{x}-0,2145 \mathrm{x}^{2}$ & 0,99 \\
\hline & Gafsa & $\hat{y}=0,1-0,811 x-0106 x^{2}$ & 0,99 & & $=5,5-2,271 \mathrm{x}-0,0627 \mathrm{x}^{2}$ & 0,99 \\
\hline & Fosbahia & $\hat{y}=1,6+0,109 x$ & 0,98 & & $=1,4+0,212 \mathrm{x}$ & 0,98 \\
\hline \multirow[t]{2}{*}{$\mathrm{V}$} & S. triplo & $\hat{y}=10,6-6,450 x-0,3323 x^{2}$ & 0,99 & $\hat{y}$ & $=11,0-1,861 \mathrm{x}-0,0271 \mathrm{x}^{2}$ & 0,99 \\
\hline & Termofosfato & $\hat{y}=0,1-1,172 \mathrm{x}$ & 0,94 & & $=6,8-1,452 \mathrm{x}-0,0284 \mathrm{x}^{2}$ & 0,94 \\
\hline
\end{tabular}

${ }^{1} \mathrm{PACd}$ : Argissolo Acinzentado distrófico; PAe: Argissolo Amarelo eutrófico; V: Vertissolo 
as demais situações, as regressões foram quadráticas (Quadro 5). Ao avaliar o efeito de nove fosfatos, Braga et al. (1991) observaram que apenas o superfosfato triplo, termofosfato e fosfato de Gafsa afetaram a produtividade de soja em quatro anos de cultivo. Mesmo com a dose $400 \mathrm{~kg} \mathrm{ha}^{-1} \mathrm{de}_{2} \mathrm{O}_{5}$, as respostas foram lineares, indicando que a dosagem de $\mathrm{P}$ foi insuficiente para obtenção da produtividade máxima.

A similaridade entre os dois extratores de $\mathrm{P}$ do solo foi observada também nas regressões com as doses de $\mathrm{P}$ aplicadas nos três solos (Quadro 6). Avaliando os valores dos coeficientes angulares das equações, constatou-se que o Mehlich-1 extraiu quantidades maiores de $\mathrm{P}$ que a resina, no PACd e PAe, principalmente quando se utilizaram o termofosfato $\mathrm{e}$ os fosfatos naturais. No Vertissolo, com exceção do Fosbahia, ocorreu o contrário, a resina extraiu mais $\mathrm{P}$ que o Mehlich-1.

A resina de troca aniônica mostrou-se mais adequada que o extrator Mehlich-1, para avaliar a disponibilidade de $\mathrm{P}$ para o arroz mantido em condições de inundação (Grande et al., 1986). Tanaka et al. (1981) observaram que o extrator Mehlich-1 apresentou maior poder de extração de $\mathrm{P}$ que os extratores $\mathrm{H}_{2} \mathrm{SO}_{4} 0,05 \mathrm{~mol} \mathrm{~L}^{-1}$ (IAC), Olsen, Bray $2 \mathrm{e}$ Bray 2 modificado, nos tratamentos com fosfato natural Patos de Minas. Ao fracionar o P, observaram que cerca de $85 \%$ correspondiam ao P-Ca e P-ocluso, tendo apenas as frações P-Al e P-Fe apresentado correlação com a resposta das plantas de milho.

Dessa forma, os dois extratores foram eficientes para avaliar a disponibilidade do $\mathrm{P}$ no solo proveniente dos fosfatos que exerceram efeito positivo na produção de matéria seca e na absorção do nutriente pelo melão. Pereira \& Faria (1978) verificaram que o método de extração por Mehlich-1 foi tão eficiente quantos os métodos Olsen e Bray 1 para avaliação do $\mathrm{P}$ aplicado sob a forma de superfosfato triplo num Vertissolo da região. Vasconcelos et al. (1986b) constataram que o Mehlich-1 apresentou-se eficiente na avaliação do $\mathrm{P}$ no solo que recebeu FN nos primeiros quatro anos de cultivo de sorgo num Latossolo Vermelho-Escuro, fase cerrado. Freitas et al. (1995) observaram que, embora o P extraído de dois solos da Zona Mata de Pernambuco pela resina de troca aniônica tenha apresentado correlação mais alta $(r=0,81)$ que a obtida por Mehlich-1 $(r=0,79)$ com o $\mathrm{P}$ absorvido pelo sorgo, os dois extratores agiram de maneira similar, apresentando uma correlação de $r=0,95$ entre os teores de $\mathrm{P}$ do solo medidos por eles. Braida et al. (1996) também concluíram que os extratores Mehlich-1 e resina de troca aniônica se equivaleram na capacidade preditiva da disponibilidade de $\mathrm{P}$ no solo para as plantas, quando os solos foram separados em classes, segundo o teor de argila.

\section{CONCLUSÕES}

1. No Vertissolo e Argissolo Acinzentado distrófico, os fosfatos apresentaram-se menos eficientes que no Argissolo Amarelo eutrófico.

2. O termofosfato foi a fonte de $\mathrm{P}$ que apresentou maior eficiência para produção de MS do melão nos

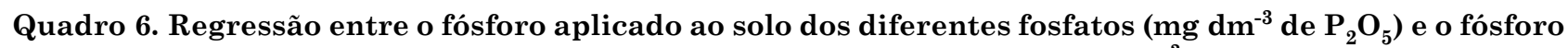
recuperado pelos extratores Melhich-1 e resina de troca aniônica $\left(\mathrm{mg} \mathrm{dm}^{-3} \mathrm{de} P\right)$

\begin{tabular}{|c|c|c|c|c|c|c|}
\hline \multirow[b]{2}{*}{ Solo(1) } & \multirow[b]{2}{*}{ Fosfato } & \multicolumn{2}{|c|}{ P - Mehlich (y) } & \multicolumn{3}{|c|}{$P$ - resina $(y)$} \\
\hline & & Equação & $\mathbf{R}^{2}$ & & Equação & $\mathbf{R}^{2}$ \\
\hline \multirow[t]{4}{*}{ PACd } & Superfosfato triplo & $\hat{y}=2,8+0,129 x$ & 0,99 & $\hat{y}$ & $=1,8+0,099 x$ & 0,99 \\
\hline & Termofosfato & $\hat{y}=1,2+0,201 \mathrm{x}$ & 0,98 & $\hat{y}$ & $=1,4+0,101 \mathrm{x}$ & 0,99 \\
\hline & Gafsa & $\hat{y}=1,6+0,485 x$ & 0,98 & $\hat{y}$ & $=1,4+0,094 \mathrm{x}$ & 0,98 \\
\hline & Fosbahia & $\hat{y}=0,9+0,410 x$ & 0,99 & $\hat{y}$ & $=2,6+0,134 \mathrm{x}$ & 0,99 \\
\hline \multirow[t]{4}{*}{ PAe } & Superfosfato triplo & $\hat{y}=3,1+0,104 \mathrm{x}$ & 0,99 & $\hat{y}$ & $=3,0+0,100 x$ & 0,96 \\
\hline & Termofosfato & $\hat{y}=1,5+0,171 x$ & 0,97 & $\hat{y}$ & $=3,6+0,081 \mathrm{x}$ & 0,98 \\
\hline & Gafsa & $\hat{y}=2,0+0,273 x$ & 0,99 & $\hat{y}$ & $=3,8+0,103 x$ & 0,98 \\
\hline & Fosbahia & $\hat{y}=3,4+0,292 x$ & 0,99 & $\hat{y}$ & $=3,0+0,150 x$ & 0,98 \\
\hline \multirow[t]{4}{*}{$\mathrm{V}$} & Superfosfato triplo & $\hat{y}=1,4+0,057 \mathrm{x}$ & 0,97 & $\hat{y}$ & $=7,0+0,135 x$ & 0,99 \\
\hline & Termofosfato & $\hat{y}=1,4+0,055 x$ & 0,98 & $\hat{y}$ & $=5,2+0,111 \mathrm{x}$ & 0,95 \\
\hline & Gafsa & $\hat{y}=2,6+0,138 x$ & 0,99 & $\hat{y}$ & $=4,2+0,251 \mathrm{x}$ & 0,98 \\
\hline & Fosbahia & $\hat{y}=2,3+0,128 \mathrm{x}$ & 0,99 & $\hat{y}$ & $=7,0+0,032 \mathrm{x}$ & 0,98 \\
\hline
\end{tabular}

(1) PACd: Argissolo Acinzentado distrófico; PAe: Argissolo Amarelo eutrófico; V: Vertissolo. 
três solos (56 a 100 \% em relação ao superfosfato triplo). No Argissolo Amarelo eutrófico, o fosfato de Gafsa também mostrou boa eficiência, sendo equivalente a $80 \%$ do superfosfato triplo.

3. Os extratores de Mehlich-1 e resina de troca aniônica foram igualmente eficientes para avaliar a disponibilidade do $\mathrm{P}$ no solo proveniente dos fosfatos, os quais exerceram efeito positivo na produção de matéria seca e absorção do nutriente pelo melão.

\section{LITERATURA CITADA}

ALMEIDA, J.C.R.; MACEDO, M.C.M. \& VALLE, C.B. Eficiência de difrentes fontes e doses de fósforo na produção de Brachiaria decumbens cv. Bsilisk em Areia Quartzosa. In: SIMPÓSIO SOBRE CERRADO, 8.; INTERNATIONAL SYMPOSIUM OF TROPICAL SAVANAS, 1., Brasília, 1996. Anais. Biodiversidade e produção sustentável de alimento e fibras nos cerrados. Planaltina, EmbrapaCPAC, 1996. p.350-353.

BEDIN, I.; FURTINI NETO, A.E.; RESENDE, A.V.; FAQUIN, V.; TOKURA, A.M. \& SANTOS, J.Z.L. Fertilizantes fosfatados e produção da soja em solos com diferentes capacidades de tampão de fosfato. R. Bras. Ci. Solo, 27:639$646,2003$.

BRAGA, N.R.; MASCARENHAS, H.A.A.; BULISANI, E.A.; RAIJ, B. van; FEITOSA, C.T. \& HIROCE, R. Eficiência agronômica de nove fosfatos em quatro cultivos consecutivos de soja. R. Bras. Ci. Solo, 15: 315-319, 1991.

BRAIDA, J.A.; CAMARGO, F.A.O.; ROSSO, I.J.; GIANELLO, C. \& MEURER, E.J. Comparação de métodos da determinação da disponibilidade de fósforo no solo para as plantas. R. Bras. Ci. Solo, 20:345-347,1996.

CHIEN, S.H. \& MENON, R.G. Factors affecting the agronomic effectiveness of phosphate rock for direct application. Fert. Res., 41:227-234, 1995.

EMPRESA BRASILEIRA DE PESQUISA AGROPECUÁRIA EMBRAPA. Centro Nacional de Pesquisa de Solos. Sistema brasileiro de classificação de solos. Brasília, Embrapa Produção de Informação; Rio de Janeiro, Embrapa Solos, 1999. 412p.

EMPRESA BRASILEIRA DE PESQUISA AGROPECUÁRIA . EMBRAPA. Centro Nacional de Pesquisa de Solos. Manual de métodos de análises de solo. 2.ed. Rio de Janeiro, 1997. 212p. (Embrapa-CNPS. Documentos,1)

FAO. Survey of the São Francisco River basin, Brazil; Soil resources and land classification for irrigation. Rome, 1966. v.2, Pte.1.

FARIA, C.M.B.; PEREIRA, J.R. \& POSSÍDIO, E.L. Adubação orgânica e mineral na cultura do melão em um Vertissolo do Submédio São Francisco. Pesq. Agropec. Bras., 29:191197, 1994.

FREITAS, A.O.S.; SABINO, S.T.B.O. \& LIMA JUNIOR, M.O Seleção de extratores de fósforo em dois solos da zona da Mata de Pernambuco. In: CONGRESSO BRASILEIRO DE CIENCIA DO SOLO, 25., Viçosa, MG, 1995. O solo nos grandes domínios morfoclimáticos do Brasil e o desenvolvimento sustentado. Resumos expandidos, Viçosa, MG, Sociedade Brasileira de Ciencia Do Solo, 1995, v.2, p.978-979.
GALRÃO, E.Z. \& VOLKWEISS, S.J. Disponibilidade de fósforo do solo para as plantas. R. Bras. Ci. Solo, 5:114-118,1981.

GOEDERT, W.J. \& LOBATO, E. Avaliação agronômica de fosfatos naturais em solo de Cerrado. R. Bras. Ci. Solo, 8:97-102, 1984.

GOEDERT, W.J. \& LOBATO, E. Eficiência agronômica de fosfatos em solos de Cerrado. Pesq. Agropec. Bras., 15:311318,1980

GRANDE, M.A.; CURI, N. \& QUAGGIO, J.A. Disponibilidade de fósforo pelos extratores de Mehlich e resina, em solos cultivados com arroz irrigado. R. Bras. Ci. Solo, 10:45-50, 1986.

LEÓN, L.A.; FENSTER, W.E. \& HAMMOND, L.L. Agronomic potential of eleven phosphate rocks from Brazil, Colombia, Peru and Venezuela. Soil Sci. Soc. Am. J., 50:798-802, 1986.

LIMA, A.A. Absorção e eficiência de utilização de nutrientes por híbridos de melão meloeiro (Cucumis melo L.). Fortaleza, Universidade Federal do Ceará, 2001. 60p. (Tese de Mestrado)

MALAVOLTA, E.; VITTI, G. C. \& OLIVEIRA, S.A. Avaliação do estado nutricional de plantas: Princípios e aplicação. 2.ed. Piracicaba, POTAFOS, 1997. 319p.

MOREIRA, A. \& MALAVOLTA, E. Fontes, doses e extratores de fósforo em alfafa e centrosema. Pesq. Agropec. Bras., 36:1519-1527, 2001.

MUNIZ, A.S.; NOVAIS, R.F.; BARROS, N.F. \& NEVES, J.C.L Nível crítico de soja na parte aérea da soja como variável do fator capacidade de fósforo do solo. R. Bras. Ci. Solo, 9:237-243, 1985.

MUTUO, P.K. Comparison of phosphate rock and triple superphosphate on a phosphorus-deficient Kenyan soil. Comm. Soil Sci. Plant Anal., 30:1091-1103, 1999.

NOVAIS, R.F. \& SMYTH, T.S. Fósforo em solo e planta em condições tropicais. Viçosa, MG, Universidade Federal de Viçosa, 1999. 399p.

OLIVEIRA, E.L.; MUZILLI, O.; IGUE, K. \& TORNERO, M.T.T. Avaliação da eficiência agronômica de fosfatos naturais. R. Bras. Ci. Solo, 8:63-67, 1984.

PEREIRA, J.R. \& FARIA, C.M.B. Avaliação de fosfatos de rocha parcialmente acidulados e tratados termicamente. In: GOEDERT, W.J. \& DIAS FILHO, F.A., eds. Relatório bienal (1986/1987). Brasília, Embrapa/PETROFERTIL, 1988. p.91-94.

PEREIRA, J.R. \& FARIA, C.M.B. Disponibilidade de fósforo aplicado em um Vertissolo do Médio São Francisco, avaliada por métodos químicos. R. Bras. Ci. Solo, 2:125128,1978

PEREIRA, J.R. \& FARIA, C.M.B. Sorção de fósforo em alguns solos do Semi-Árido do Nordeste Brasileiro. Pesq. Agropec. Bras., 33:1179-1184, 1998.

PRATA, E.B. Acumulação de biomassa e absorção de nutrientes por híbridos de meloeiro (Cucumis melo L.). Fortaleza, Universidade Federal do Ceará, 1999. 61p. (Tese de Mestrado) 
RAIJ, B. van; ANDRADE, J.C.; CANTARELLA, H. \& QUAGGIO, J.A., eds. Analise química para avaliação da fertilidade de solos tropicais. Campinas, Instituto Agronômico de Campinas, 2001. 285p.

SANZONOWICZ, C. \& GOEDERT, W.J. Uso de fosfatos naturais em pastagens. Planaltina, Embrapa-CPAC, 1986. 33p. (Embrapa-CPAC. Circular Técnica, 21)

SANZONOWICZ, C.; LOBATO, E. \& GOEDERT, W.J. Efeito residual da calagem e de fontes de fósforo numa pastagem estabelecida em solo de Cerrado. Pesq. Agropec. Bras., 22:233-243, 1987.

SILVA, F.C. \& RAIJ, B.van Disponibilidade de fósforo em solos avaliada por diferentes extratores. Pesq. Agropec. Bras., 34:267-288, 1999.

SNEDECOR, G.W. \& COCHRAN, W.G. Métodos estatísticos. México, Continental, 1971. 703p.

SYERS, J.K.; MACKAV, A.D.; BROWN, M.W. \& CURRIE, L.D. Chemical and physical characteristics of phosphate rock materials of varying reactivity. J. Sci. Food Agric., 37:10571064, 1986.
TANAKA, R.T.; BAHIA, V.G.; COELHO, A.M. \& FREIRE, J.C. Seleção de extratores de fósforo do solo em função das respostas de milho (Zea mays L.) e da adubação com fosfato patos de minas em condições de casa de vegetação. R. Bras. Ci. Solo, 5:38-42, 1981.

VASCONCELLOS, C.A.; SANTOS, H.L.; FRANCA, G.E.; PITTA, G.V.E. \& BAHIA FILHO, A.F.C. Eficiência agronômica de fosfatos naturais para a cultura do sorgogranífero. I. Fósforo total e solúvel em ácido cítrico e granulometria. R. Bras. Ci. Solo, 10:117-121, 1986a.

VASCONCELlOS, C.A.; SANTOS, H.L.; FRANCA, G.E.; PITTA, G.V.E. \& BAHIA FILHO, A.F.C. Eficiência agronômica de fosfatos naturais para a cultura do sorgogranifero. II. Produção de grãos, eficiência relativa e fósforo disponível. R. Bras. Ci. Solo, 10:123-128, 1986b.

YOST, R.S.; NADERMAN, G.C.; KAMPRATH, E.J. \& LOBATO, E. Availability of rock phosphate as measured by an acid tolerant pasture grass and extractable phosphorus. Agron. J., 74:463-468, 1982.

ZOYSA, A.K.N. Phosphorus utilization efficiency and depletion of phosphate fractions in the rhizosphere of three tea (Camellia sinensis L.) clones. Nutr. Cycling Agroecosys., 53:189-201, 1999. 